\title{
Cutting Depth Dictates the Transition from Continuous to Segmented Chip Formation
}

\author{
Ramin Aghababaei® ${ }^{1,2, *}$ Mohammad Malekan ${ }^{1,3}$ and Michal Budzik $\oplus^{1,2}$ \\ ${ }^{1}$ Department of Mechanical and Production Engineering, Aarhus University, 8000 Aarhus C, Denmark \\ ${ }^{2}$ Centre for Integrated Materials Research (iMAT), Aarhus University, 8000 Aarhus C, Denmark \\ ${ }^{3}$ SDU Mechatronics, Department of Mechanical and Electrical Engineering, University of Southern Denmark, \\ 6400 Sønderborg, Denmark
}

(Received 13 July 2021; accepted 20 October 2021; published 3 December 2021)

\begin{abstract}
The process of material cutting emerges from a series of nonlinear phenomena including frictional contact, plastic deformation, and fracture. While cutting dominated by shear deformation is of interest to achieve a smooth material removal and a high-quality surface finish, the fracture-induced chip breaking is of equal importance to prevent the formation of long chips. Here we show that discrepant observations and predictions of these two distinct cutting mechanisms can be reconciled into a unified framework. A simple analytical model is developed to predict the mechanism of chip formation in a homogeneous medium as a function of work piece intrinsic material properties, tool geometry, and the process parameters. The model reveals the existence of a critical depth of cut, below which the chip formation is gradually progressed by plastic deformation in the shear plane, and above which chips break off by abrupt crack propagation. The models' prediction is validated by systematic in situ orthogonal cutting experiments and literature data for a wide range of materials over multiple length scales.
\end{abstract}

DOI: 10.1103/PhysRevLett.127.235502

The process of chip formation during cutting starts with the elastic deformation of surface material followed by severe plastic shearing or fracture. In ductile materials, the material ahead of the cutting edge is plastically deformed, forming a shear band zone which smoothly separates the chip into lamella of nearly uniform thickness [1], referred to as the shear cutting (SC) [2-5] process [Fig. 1(a)]. For brittle solids, chips break off as a result of abrupt subsurface cracking, leaving rough marks on the surface, referred to as the fracture cutting (FC) [2-5] process [Fig. 1(b)]. Cutting experiments and simulations [6-9] over the past decade confirmed that the material removal mechanism is a function of several factors including depth of cut (DoC) [4,5,7,10-13], cutting environment (e.g., temperature, speed, and lubrication) $[14,15]$, tool material, and geometry $[11,16]$.

Classical mechanistic theories of cutting [17-21] describe the material flow and ploughing as the main mechanism of material removal. Analysis by Akono et al. $[2,22]$ suggested that, when the cutting width is much bigger than the work piece fracture process zone size and the ratio between the cutting width and depth gets bigger than 2 , the process can be described via the linear elastic

Published by the American Physical Society under the terms of the Creative Commons Attribution 4.0 International license. Further distribution of this work must maintain attribution to the author(s) and the published article's title, journal citation, and DOI. fracture mechanics. For a constant cutting width, their analysis suggests that the process of material removal is dominated by fracture when the cutting depth is smaller than a certain value. Two recent study [5,13], however,
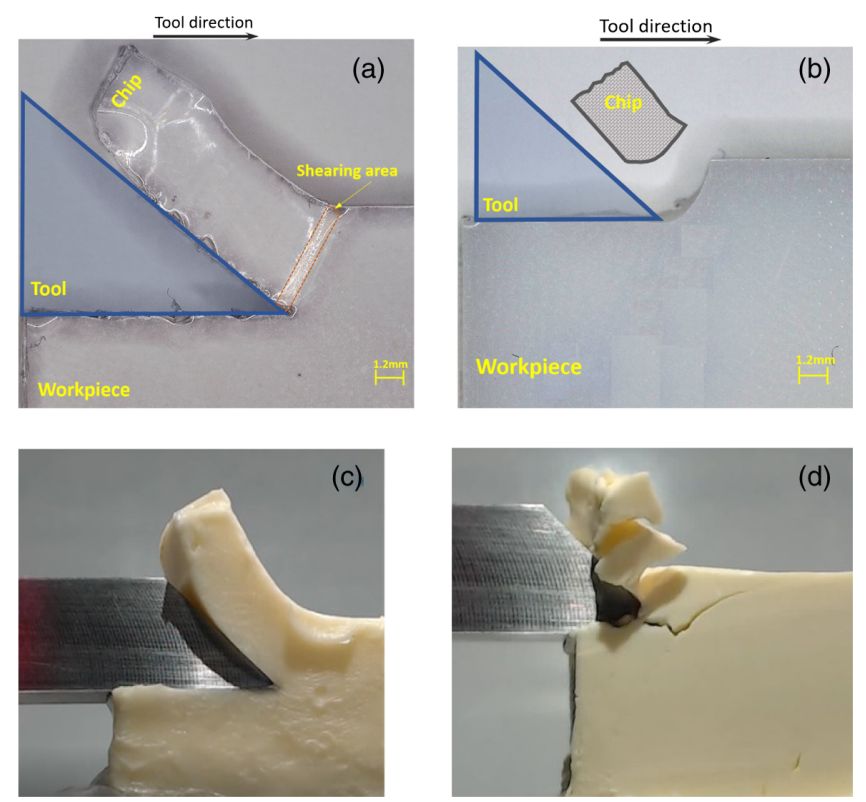

FIG. 1. Representative snapshots of our cutting experiments, illustrating (a) the gradual chip formation by plastic shearing in $\mathrm{PC}$ at $4 \mathrm{~mm}$ DoC and (b) fracture-induced abrupt chip breakage in POM at $2.5 \mathrm{~mm}$ DoC. (c),(d) The shear- versus fracture-induced material removal mechanisms in butter at the room temperature $\left(17^{\circ}\right)$ and freezing temperature of $-10^{\circ}$, respectively. 
identified experimentally a critical DoC, below which brittle solids undergo microscopic plastic shearing resulting in a crack-free surface. These discrepant results raise a question of how and to what extent individual material properties and tool geometry control the mechanism of chip formation and eventual material removal.

Here, we focus on the influence of DoC on the transition in the chip formation mechanism in a homogeneous medium through a combination of theoretical analysis and controlled cutting experiments. We performed orthogonal cutting tests systematically varying the DoC on three different polymers [polymethyl methacrylate (PMMA), polyoxymethylene (POM), and polycarbonates (PC)] with very distinct material properties (i.e., brittle and ductile). Representative snapshots of our experiments, illustrating the process of shear cutting in PC and the chip breakage in POM, are presented in Figs. 1(a) and 1(b), respectively. To further visualize these two chip formation mechanisms and the effect of environmental conditions, we also performed two additional tests cutting through butter at room temperature $17^{\circ}$ [Fig. 1(c)] and the same butter frozen at $-10^{\circ}$ [Fig. 1(d)] while keeping the DoC constant at $9 \mathrm{~mm}$.

To find the depth at which the transition in chip detachment mechanism occurs, we derive and compare cutting forces necessary to initiate individual chipping mechanisms at steady-state cutting, i.e., plastic shearing versus chip fracture. To compute the force for detaching a chip by subsurface crack propagation toward the free surface [Fig. 2(a)], we use the linear elastic fracture mechanics considering a double cantilever beam geometry $[23,24]$. Accordingly, the energy release rate $G$ can be obtained as

$$
G=\frac{1}{E w^{2} d}\left(N^{2}+S^{2}+\frac{12 M^{2}}{d^{2}}\right),
$$

where $E$ is the elastic modulus, $w$ is the out-of-plane width, and $d$ is the chip thickness. $N, S$, and $M$ represent the magnitude of the normal and shear forces and the bending moment at the crack tip [see Fig. 2(a)]. Ignoring the friction force between the chip and the tool rake face, the forces and moment can be substituted as $M=F_{n} h, S=F_{n}$, and $N=0$ in Eq. (1), where $F_{n}$ represents the external force normally applied to the chip edge by the tool and $h$ is the tool-chip contact length. Assuming the plane stress condition $\left(K_{I C}=\sqrt{G E}\right)$, the force at the onset of chip detachment by crack propagation, $F_{f r}$, can be obtained as

$$
F^{f r}=F_{n} \cos \lambda=K_{I C} w \cos \lambda \sqrt{d} \frac{1}{\sqrt{12 \alpha^{2}+1}},
$$

where $\lambda$ is the rake angle of the cutting tool and $\alpha=h / d$. Previous experiments and theoretical analysis [25-27] showed that the tool-chip contact length scales proportionally with the chip thickness with a proportionality factor $\alpha$ between 1 and 2 [26-28].
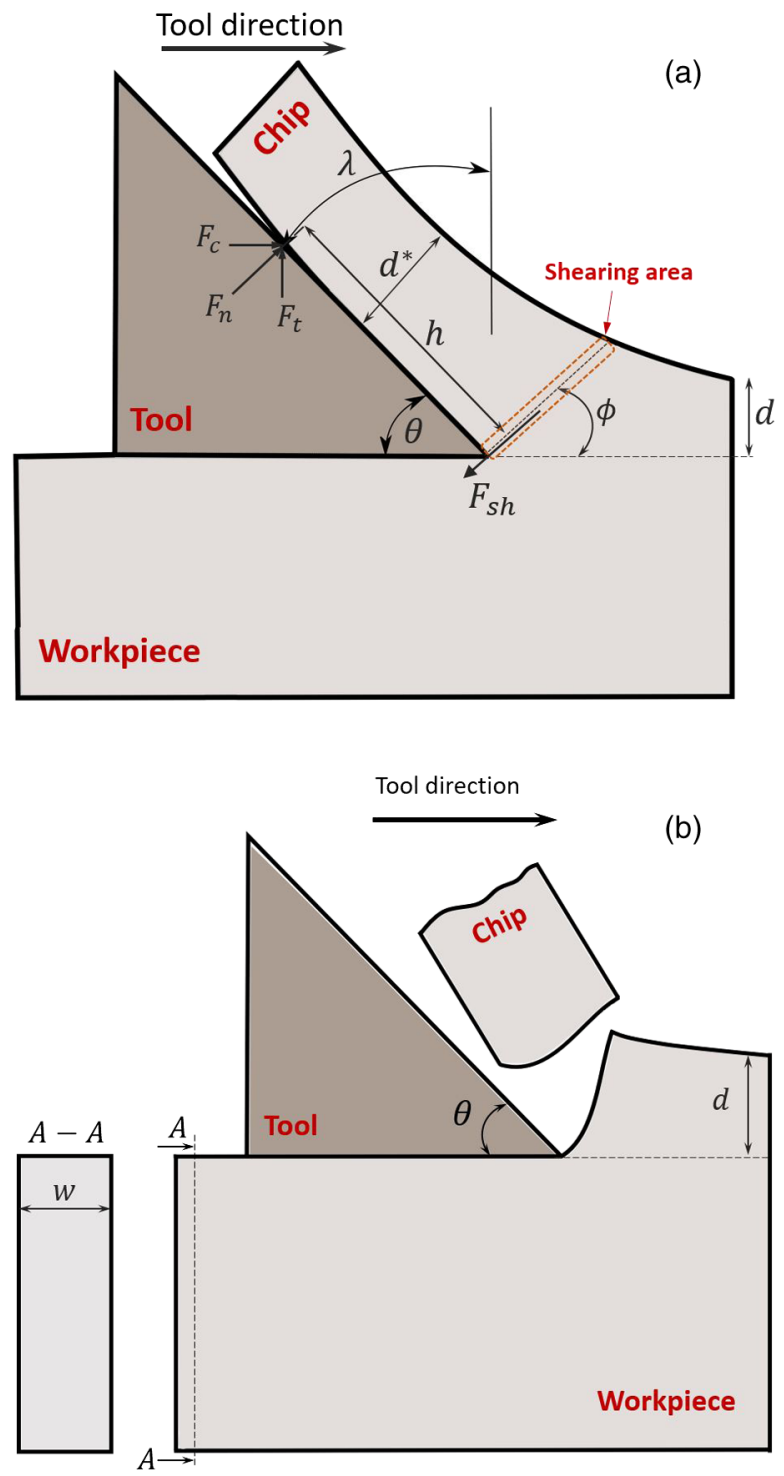

FIG. 2. Illustration of two material removal chipping mechanisms: (a) Gradual chip formation progressed by plastic shearing and (b) fracture-induced chip breakage.

It should be noted that Eq. (2) predicts the fracture force in the presence of a sharp crack tip, i.e., $\theta=0$, where the linear elastic fracture mechanics approach assumes a square root stress singularity. We show in Appendix A of the Supplemental Material [29] that this solution can also provide a good estimation of fracture force for crack tip angle below $90^{\circ}$.

The force required to progress the material removal via plastic shearing on the primary shear zone, in which plastic deformation spreads from the tip of the tool to the material surface [30,31], can be obtained as [31]

$$
F^{\mathrm{pl}}=F_{\mathrm{sh}} \cos \phi=\frac{w \tau_{y s} d \cos \phi}{\sin \phi},
$$

where $F_{\text {sh }}$ is the force causing plastic shearing along the primary shear plane and $\tau_{y s}$ is the material shear strength. $\phi$ 

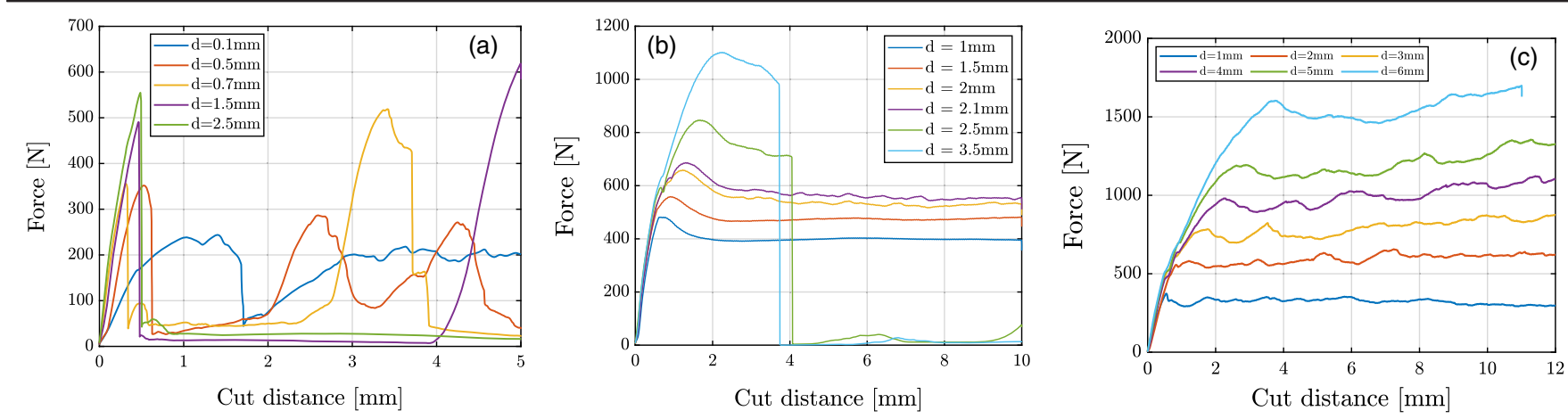

FIG. 3. Cutting force-distance curves under different DoCs for (a) PMMA with $d=0.1-2.5 \mathrm{~mm}$, (b) POM with $d=1-3.5 \mathrm{~mm}$, and (c) PC with $d=1-6 \mathrm{~mm}$. The corresponding chip formation mechanism can be seen in Supplemental Material [29] Fig. S3 and supplemental movies.

represents the inclination angle of the shear plane from the cutting velocity vector, measured on the orthogonal plane, i.e., shear plane angle [see Fig. 2(a)]. Using Merchant's equation [32], the shear plane angle can be written as a function of the tool rake angle $(\lambda)$ and friction angle $(\beta), \phi=\pi / 4+\lambda / 2-\beta / 2$.

Comparing the variation of the force for the fractureinduced chip breakage [Eq. (2)] and chip removal progression by plastic shearing [Eq. (3)] as a function of DoC, one can find a critical DoC, below and above which the gradual plastic shearing and abrupt chip breakage are the dominant material removal mechanism

$$
d_{c}=\underbrace{\frac{(\tan \phi \cos \lambda)^{2}}{12 \alpha^{2}+1}}_{\text {shape factor }}\left(\frac{K_{I C}}{\tau_{y s}}\right)^{2} .
$$

This equation highlights that the transition in chipping mechanism is a function of both material and geometrical factors, suggesting that the critical DoC is a system characteristic rather than a material constant.

To validate Eq. (4), we conducted a systematic set of orthogonal cutting experiments, varying the depth of cut for three different polymers (PMMA, POM, and PC). The setup simulates the process of chip formation by cutting through plates with out-of-plane width of 4,5 , and $5 \mathrm{~mm}$ for PMMA, POM, and PC, respectively.

The cutting tool was manufactured from S355 steel with cutting angle of $45^{\circ}$ and tip radius of $R=0.05 \mathrm{~mm}$. The cutting setup is shown in Appendix C of the Supplemental Material [29] (Fig. S2) where the cutting edge cut through work piece plates with a constant speed of $20 \mathrm{~mm} / \mathrm{min}$, while the cutting force is measured via a load censor with a maximum limit of $2 \mathrm{kN}$. Selected snapshots and movies of some experiments can be find in Supplemental Material Fig. S3 and videos [29], respectively.

Figure 3 presents variation of the cutting force for PMMA, POM, and PC for different DoC. In agreement with the prediction of Eq. (4), Fig. 3(a) shows that for
PMMA, down to the cutting depth of $0.5 \mathrm{~mm}$, the material removal is dominated by fracture-induced chip breakage, indicated by a sudden drop in the cutting force, seen in Supplemental Material [29], Figs. S3(a) and S3(b). For $0.1 \mathrm{~mm}$ cutting depth, which is the smallest possible cutting depth in our setup, a large degree of ductility and plastic shearing can be observed in Fig. 3(a) [see also Supplemental Material [29], Figs. S3(a)].

The transition in material removal mechanisms is more clearly visible from cutting force-distance curves of POM in Fig. 3(b) and Supplemental Material [29], Figs. S3(c) and S3(d). As seen, the cutting force reaches a steady state for DoC up to $2.1 \mathrm{~mm}$, indicating the gradual chip removal by plastic shearing. Alternatively, the material removal progresses by chip breakage for larger DoCs (i.e., 2.5 and $3.5 \mathrm{~mm}$ ), resulting in an abrupt drop of cutting force to zero.

Limited by the maximum load capacity of our cutting setup, we only observed one regime of cutting in PC [Fig. 3(c) and Supplemental Material [29], Fig. S3(e)], i.e., steady state gradual material removal by plastic shearing, up to $6 \mathrm{~mm}$ DoC. The removal by plastic shearing can be further justified by noting that the cutting force increases linearly with DoC [see Eq. (3) and Supplemental Material [29] Fig. S4]. Supplemental Material Fig. S5 [29] further inspects the mechanism of material removal by microscopic analysis of surface finish, comparing the ductile versus brittle fracture.

Figure 4 compares the prediction of Eq. (4) with our test results (PMMA, POM, and PC) as well as available test data from previous research for a wide range of materials and cutting conditions over multiple length scales. Comparing Eq. (4) with our experimental data suggests the chip length-thickness ratio of $\alpha=1.2$ and the critical DoC of 0.14, 2.16, and 5.2 mm for PMMA, POM, and PC, respectively. Material properties of tested polymers, used for this calculation, are listed in Appendix B of the Supplemental Material, Table S1 [29]. Additionally, we consider a rake angle of $45^{\circ}$ and an assumed friction coefficient of $\mu=0.3$. 


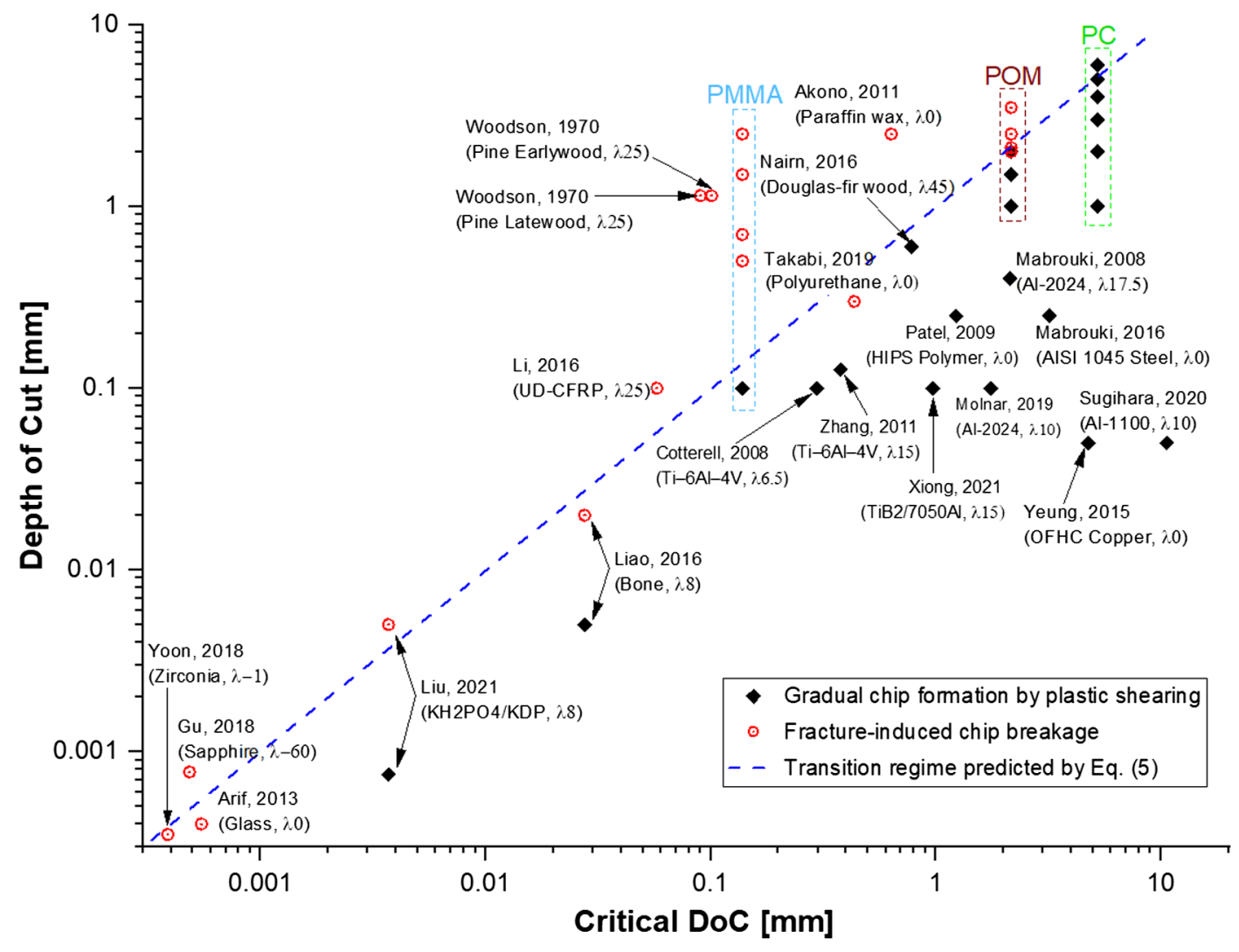

FIG. 4. A compilation of our cutting experiments on PMMA, POM, and PC as well as cutting data from previous studies $[2,3,5,9,11,16,21,33-43]$. This comparison highlights two distinct mechanisms of material removal and chip formation: Gradual chip removal by plastic shearing and fracture-induced abrupt chip breakage. It can be seen that the developed model in this study [Eq. (4)] can fairly predict the transition in material removal mechanisms for a wide range of materials over multiple scales. The critical DoC for different materials has been calculated using material properties listed in the Supplemental Material, Table S2, with the rake angle of $45^{\circ}$, coefficient of friction $\mu=0.3$ and $\alpha=1.2$. $\lambda \#$ shows the value of the tool rake angle used in those experiments.

To evaluate the accuracy of Eq. (4) to predict the chip removal mechanism, we superimpose additional experimental data from previous researches into Fig. 4, using the same friction coefficient of $\mu=0.3$ and $\alpha=1.2$. We only consider experiments with positive rake angle for which the assumption of linear elastic fracture mechanics in Eq. (2) is valid for the fracture-induced chip breakage. Inserting values for the material shear strength and critical fracture toughness (detailed in, Table S2 [29]) with the corresponding rake angle into Eq. (4), it can be seen that the proposed model predicts remarkably the operative material removal mechanisms for most cases. As seen, Fig. 4 divides the experimental data into two distinct cutting regimes, i.e., abrupt fracture-induced chip breakage and gradual chip formation by plastic shearing where the transition occurs at a critical DoC.

The ability of the model to predict the transition in chip removal mechanisms, not only confirms the existence of a critical DoC, but explains discrepant experimental observations. The absence of a plastic shearing mechanism in Akono et al. [2] experiments with paraffin wax can be explained as the range of cutting depth in their experiments
$(2.5-25 \mathrm{~mm})$ is larger than the critical DoC $(<1 \mathrm{~mm})$, predicted by Eq. (4) for paraffin wax. Our results suggest that for typical cutting applications, where the cutting depth is usually smaller than the cutting width, the mechanism of material removal and chip formation is dictated by the cutting depth. In order to use Eq. (4) for describing the scratching process, in which the cutting depth and width are comparable, an additional constraint related to the width dimension may need to be taken into account.

Furthermore, Eq. (4) suggests that for a certain material and cutting parameters (i.e., DoC and cutting edge geometry), the chipping mechanism can be tuned by modifying the critical DoC via changing environmental conditions, e.g., running temperature. It is known that for the majority of materials, while the shear strength decreases, the ductility increases with temperature, resulting in a higher critical DoC [see Eq. (4)]. This is confirmed by our simple cutting experiments with butter, in which the gradual chip formation at the room temperature [Fig. 2(c)] and the fracture-induced chip breakage at temperature $-17^{\circ}$ [Fig. 2(d)] are observed while keeping DoC constant. 
Figure 4 presents experimental data mainly for ceramics and a few metals where the critical DoC is at the submillimeter range. Equation (4) suggests that the critical DoC increases quadratically by increasing the $K_{I C}$ and decreasing the $\tau_{y s}$. As a result, a larger DoC range (i.e., centimeter scale) and resultant cutting forces are required to observe a transition in ductile metals such as copper and aluminium. This explains the limited amount of studies investigating the fracture-induced chip formation in ductile metals.

This study has a potential application for designing cutting tools and optimizing manufacturing processes by combining a right set of material and process parameters. It opens also new directions for computational and experimental mechanics as well as material science communities to further investigate the effect of other geometrical parameters (e.g., cutting edge nose radius, oblique cutting angle), internal microstructures and inhomogeneities (e.g., voids, cracks, particles, dislocations, grain boundaries) on the removal mechanism. It is also of interest to study the transition from cutting to scratching processes and relevant models $[41,44]$.

R. A. and M. M. acknowledge financial support from the Innovation Fund Denmark (Innovationsfonden) for the project Cutting Edge, 8090-00010B. The authors thank Mr. Imre Toth for designing and partially conducting the cutting tests. The authors also acknowledge useful discussion with Mr. Klaus Bonde $\varnothing$ rskov from the Danish Advanced Manufacturing Research Center.

* Corresponding author. ra@mpe.au.dk

[1] C. Arcona and T. A. Dow, J. Manuf. Sci. Eng. 120, 700 (1998).

[2] A.-T. Akono, P. M. Reis, and F.-J. Ulm, Phys. Rev. Lett. 106, 204302 (2011).

[3] Z. Liao and D. Axinte, Int. J. Mach. Tools Manufact. 102, 41 (2016).

[4] O. Diaz and D. Axinte, Int. J. Mach. Tools Manufact. 118119, 12 (2017).

[5] Q. Liu, Z. Liao, J. Cheng, D. Xu, and M. Chen, Mater. Des. 198, 109327 (2021).

[6] A. Vandana and N. Sundaram, Tribol. Lett. 66, 94 (2018).

[7] G. Xiao, M. Ren, and S. To, Micromachines 9, 49 (2018).

[8] Y. Xiong, W. Wang, R. Jiang, K. Lin, and M. Shao, Materials 11, 606 (2018).

[9] B. Takabi and B. Tai, J. Manuf. Mater. Process. 3, 36 (2019).

[10] J. Zhou, M. Andersson, and J. Stahl, Int. J. Adv. Manuf. Technol. 22, 697 (2003).

[11] T. G. Molnar, S. Berezvai, A. K. Kiss, D. Bachrathy, and G. Stepan, Int. J. Mach. Tools Manufact. 145, 103429 (2019).
[12] W. Liu, X. Zhu, and J. Jing, J. Pet. Sci. Eng. 163, 311 (2018).

[13] A. Singh, D. Solanki, R. Sencha, R. K. Singh, R. G. Mote, and R. K. Singh, J. Manufact. Proc. 58, 749 (2020).

[14] R. W. Maruda, G. M. Krolczyk, P. Nieslony, S. Wojciechowski, M. Michalski, and S. Legutko, J. Manuf. Proc. 24, 107 (2016).

[15] A. Li, J. Zhao, and G. Hou, Adv. Mech. Eng. 9(7), 1 (2017).

[16] Y. Zhang, T. Mabrouki, D. Nelias, and Y. Gong, Finite Elements in Analysis and Design 47, 850 (2011).

[17] M. E. Merchant, J. Appl. Phys. 16, 318 (1945).

[18] M. E. Merchant, J. Appl. Phys. 16, 267 (1945).

[19] N. K. Sundaram, Y. Guo, and S. Chandrasekar, Phys. Rev. Lett. 109, 106001 (2012).

[20] A.-T. Akono, F.-J. Ulm, and Z. P. Bazant, Eng. Fract. Mech. 119, 21 (2014).

[21] H. Yeung, K. Viswanathan, W. D. Compton, and S. Chandrasekar, Proc. Natl. Acad. Sci. U.S.A. 112, 9828 (2015).

[22] A.-T. Akono and F.-J. Ulm, J. Mech. Phys. Solids 60, 379 (2012).

[23] Z. Suo and J. Hutchinson, Int. J. Fract. 43, 1 (1990).

[24] H. Jensen and M. Thouless, Int. J. Solids Struct. 30, 779 (1993).

[25] E. Lee and B. Shaffer, ASME J. Appl. Mech. 18, 405 (1951).

[26] S. Iqbal, P. Mativenga, and M. Sheikh, Int. J. Adv. Manuf. Technol. 42, 30 (2009).

[27] A. Fatima and P. Mativenga, Proc. Inst. Mech. Eng. B J. Eng. Manuf. 227, 345 (2013).

[28] L. H. Huang, J. C. Chen, and T. Chang, J. Indust. Technol. 15(2), 1 (1999), https://cdn.ymaws.com/atmae.site-ym.com/ resource/resmgr/JIT/huan0399.pdf.

[29] See Supplemental Material at http://link.aps.org/ supplemental/10.1103/PhysRevLett.127.235502 for a detailed procedure for developing the theoretical model in Appendix A, material properties of tested polymers, and materials adopted from the literature in Fig. 4 in Appendix B, and details of cutting experiments setup in Appendix C.

[30] J. Williams, Y. Patel, and B. Blackman, Eng. Fract. Mech. 77, 293 (2010)

[31] J. Williams and Y. Patel, Interface Focus 6, 20150108 (2016).

[32] H. Ernst and M. E. Merchant, Trans. ASME 29, 1 (1941).

[33] G. Woodson and P. Koch, U.S. Department of Agriculture. Forest Service Research Paper SO-52 (1970).

[34] T. Mabrouki, F. Girardin, M. Asad, and J.-F. Rigal, Int. J. Mach. Tools Manufact. 48, 1187 (2008).

[35] M. Cotterell and G. Byrne, CIRP J. Manufact. Sci. Technol. 1, 81 (2008).

[36] Y. Patel, B. Blackman, and J. Williams, Eng. Fract. Mech. 76, 2711 (2009).

[37] M. Arif, Z. Xinquan, M. Rahman, and S. Kumar, Int. J. Mach. Tools Manufact. 64, 114 (2013).

[38] T. Mabrouki, C. Courbon, Y. Zhang, J. Rech, D. Nlias, M. Asad, H. Hamdi, S. Belhadi, and F. Salvatore, C.R. Mec. 344, 335 (2016).

[39] J. Nairn, Inter. Focus 6, 20150110 (2016).

[40] H.-S. Yoon, S. Lee, and S. Min, Proc. Manufact. 26, 443 (2018). 
[41] X. Gu, H. Wang, Q. Zhao, J. Xue, and B. Guo, Int. J. Mech. Sci. 148, 565 (2018).

[42] T. Sugihara, A. Udupa, K. Viswanathan, J. M. Davis, and S. Chandrasekar, Sci. Adv. 6 (2020).
[43] Y. Xiong, W. Wang, Y. Shi, R. Jiang, C. Shan, X. Liu, and K. Lin, Chinese Journal of aeronautics 34, 451 (2021).

[44] M. Arif, M. Rahman, and E. Y. San, Int. J. Mach. Tools Manufact. 51, 170 (2011). 